\title{
Renal findings in rheumatoid arthritis: clinical aspects of 132 necropsies
}

\author{
MAARTEN BOERS, ${ }^{1}$ ANITA M CROONEN, ${ }^{2}$ BEN A C DIJKMANS, \\ FERRY C BREEDVELD,${ }^{1}$ FRITS EULDERINK, ${ }^{2}$ ARNOLD CATS, ${ }^{1}$ AND \\ JAN J WEENING
}

From the 'Department of Rheumatology, Geb. I, C2-Q and the ${ }^{2}$ Department of Pathology, Leiden University and University Hospital, PO Box 9600, 2300 RC Leiden, The Netherlands

SUMMARY Renal abnormalities in 132 necropsied patients with rheumatoid arthritis were $\frac{\vec{\sigma}}{\circ}$ studied. Clinical findings before death included extra-articular manifestations of the disease 6 (86\% of patients), systemic vasculitis $(6 \%)$, and uraemia $(23 \%)$. Necropsy findings included $\circlearrowleft$ nephrosclerosis $(90 \%)$, systemic vasculitis $(14 \%)$ with kidney involvement in $8 \%$, amyloidosis 0 $(11 \%)$, membranous glomerulopathy $(8 \%)$, and focal glomerular disease $(8 \%)$. Association with clinical data suggests that both rheumatoid and non-rheumatoid disease may play a part in the $\mathcal{\infty}$ cause of these abnormalities.

Key words: vasculitis, amyloidosis, proliferative glomerulonephritis.

Renal involvement in rheumatoid arthritis (RA) is well recorded. Involvement leading to loss of renal function is mainly attributed to the development of amyloidosis or to adverse reactions to drug treatment, ${ }^{1-5}$ and in a few cases to rheumatoid vasculitis affecting the renal vessels. ${ }^{67}$ Several necropsy and biopsy studies have shown non-specific kidney changes, which were ascribed to RA. The lesions included non-specific changes such as mesangial hypercellularity, ${ }^{8-16}$ nephrosclerosis, ${ }^{8} 131417-19$ and the incidental finding of proliferative glomerulonephritis $^{2021}$ or membranous glomerulopathy ${ }^{22}$ attributable only to the rheumatoid disease. It is not certain, however, whether these lesions must be considered a part of the rheumatoid disease because the association was not found by all of the authors, ${ }^{1723-26}$ and disease other than RA underlying the renal abnormalities cannot always be ruled out.

The aim of the present study was to evaluate the renal abnormalities in a large series of necropsied patients with RA and to detect correlations between these findings and possibly predictive clinical data. Amyloidosis, membranous glomerulopathy, proliferative glomerulonephritis, and renal vasculitis, but also non-specific changes in glomeruli, blood

Accepted for publication 13 April 1987.

Correspondence to Dr Maarten Boers, Department of Rheumatology. Geb. I, C2Q, Leiden University and University Hospital, PO Box 9600,2300 RC Leiden. The Netherlands. vessels, and the interstitium, were present in significant proportion of our patients. The occur rence of some of these findings was shown to related to clinical aspects of RA and concomitant (non-rheumatic) disease.

\section{Patients and methods}

The series comprises all cases of classical or 3 definitive RA treated and necropsied in our hospital between 1958 and 1984. Hospital charts were? reviewed to collect the following data relevant to RA and to renal disease: sex, age, duration of RA, $\stackrel{2}{\circ}$ extra-articular manifestations, treatment of RA, 3 presence of hypertension (defined as untreated diastolic pressure higher than $100 \mathrm{mmHg}$ ), diabetes $\frac{}{3}$ mellitus requiring therapy, symptomatic arteriosclerosis (leading to ischaemia or infarction),,$\frac{9}{9}$ recurrent urinary infection (two or more infections? in two consecutive years), the presence (at any time) of serum rheumatoid and antinuclear factors, urine $N_{O}$ analysis data and serum creatinine levels in the yearpreceding the final hospital admission, and last 0 values obtained before death. For descriptivew purposes loss of renal function was assumed when? serum creatinine exceeded $100 \mu \mathrm{mol} / \mathrm{l}$ and uraemia at serum levels above $200 \mu \mathrm{mol} / \mathrm{l}$.

Necropsy reports were studied with special $\stackrel{\infty}{+}$ attention to renal abnormalities. For each patient, $\underline{T}$ sections of paraffin embedded kidney tissue were $\frac{\overrightarrow{\mathbb{D}}}{\mathrm{D}}$ 
reviewed by two pathologists without knowledge of the clinical data. Only light microscopy was performed. Glomerular lesions were classified according to the WHO criteria. ${ }^{27}$ Non-specific lesions of benign nephrosclerosis, including vascular and glomerular sclerosis, were scored on an arbitrary scale as absent, mild, moderate, or severe. Nonspecific changes in tubuli and interstitium, including atrophy and fibrosis, were scored separately, even though these lesions are closely associated with benign nephrosclerosis.

\section{STATISTICAL ANALYSIS}

Median and range were used to summarise the frequency distribution of most variables. Clinical and necropsy results were compared on the basis of contingency tables, and $\chi^{2}$ tests were used to detect statistically significant associations. The number of missing cases for a given variable did not exceed 10 unless stated otherwise.

\section{Results}

C L I N ICA L DATA

The analysis covered 132 patients (35 out of 167 were excluded because of insufficient clinical data or pathological data, or both). The necropsy rate for patients with RA was stable throughout the study period at approximately $70 \%$. A median number of eight cases (range 1-11) was added to the series each year.

There were 73 female $(55 \%)$ and 59 male patients $(45 \%)$ and the median age was 65 years (range 35-86 years). Median duration of RA was 14.5 years (range 1-51 years). One hundred and nine patients $(86 \%)$ had one or more extra-articular manifestations of RA (median 2; range 0-9) (Table 1). Thirty eight patients $(29 \%)$ had hypertension, 14 $(11 \%)$ had diabetes mellitus, $39(30 \%)$ had arterio-

Table 1 Extra-articular manifestations of rheumatoid arthritis in 132 necropsied patients*

\begin{tabular}{ll}
\hline Symptom/sign & No of patients $(\%)$ \\
\hline Nodules & $65(50)$ \\
Malaise/weight loss $(>5 \mathrm{~kg})$ & $60(47)$ \\
Pleuro/pericarditis, valve lesions & $41(32)$ \\
Skin ulcers/purpuric lesions & $29(22)$ \\
Spleen enlargement & $15(12)$ \\
Neuro/myopathy & $15(12)$ \\
Sjögren's syndrome & $9(7)$ \\
Eye symptoms & $8(6)$ \\
Nail fold lesions & $8(6)$ \\
Felty's syndrome & $7(5)$ \\
Vasculitis (in biopsy material) & $6(4)$ \\
\hline
\end{tabular}

${ }^{*}$ Some of the patients had two or more of the signs or symptoms.
Table 2 Macroscopic findings in the kidneys of 132 necropsied patients with rheumatoid arthritis

\begin{tabular}{ll}
\hline Findings & No of patients $(\%)$ \\
\hline Malformation & $13(10)$ \\
End stage kidney & $11(8)$ \\
Tumour (including metastases) & $9(7)$ \\
Stones/sludge & $7(5)$ \\
Infarction & $5(4)$ \\
Hydroncphrosis & $4(3)$ \\
Papillary necrosis & $2(2)$ \\
Hacmorrhage & $2(2)$ \\
Perinephritic abscess & $1(1)$ \\
\hline
\end{tabular}

*Some of the patients had two or more of the listed phenomena.

sclerosis, and $30(23 \%)$ had recurrent urinary infections. Seventy seven patients $(58 \%)$ had at least one of these diseases.

Rheumatoid factors were found in 112 patients $(89 \%)$, antinuclear factors in $59(61 \%, 96$ patients tested). In the final year of their illness 26 patients $(20 \%)$ had urine abnormalities (predominantly proteinuria) together with normal renal function, and $28(21 \%)$ had urine abnormalities with loss of renal function (as defined in 'Patients and methods'); 14 patients $(11 \%)$ had loss of renal function with normal urine analysis findings. Thirty one patients $(23 \%)$ were uraemic at death.

All of the patients had been treated with several non-steroidal anti-inflammatory drugs during the course of the RA. Most of the patients had probably used phenacetin compounds, but regular use was reported for only $23(17 \%)$. Ninety patients $(73 \%)$ had received antimalarials, $60(48 \%)$ systemic steroids, and $13(10 \%)$ azathioprine. No renal side effects of these drugs were noted. One hundred patients $(79 \%)$ were treated with gold; 16 of them had developed renal side effects (usually proteinuria). Fifteen patients $(11 \%)$ were treated with D-penicillamine, and five of them had developed renal side effects.

NECROPSY AND RENAL TISSUE REVIEW

General arteriosclerosis was observed at necropsy in 77 patients $(58 \%) ; 49(37 \%)$ showed recent or scarred infarction of one or more organs. Macroscopic abnormalities were found in the kidneys of 43 patients (33\%) (Table 2).

\section{Renal amyloidosis}

Renal amyloidosis was found in 14 patients $(11 \%)$; there were no cases of amyloidosis without renal involvement. One case showed concomitant focal glomerulonephritis; another showed concomitant systemic vasculitis without renal involvement. 
Table 3 Renal histology in 18 patients with rheumatoid arthritis and systemic vasculitis at necropsy*

\begin{tabular}{lccc}
\hline $\begin{array}{l}\text { Vasculitis in large } \\
\text { renal vessels }\end{array}$ & \multicolumn{3}{c}{ Proliferative } \\
\cline { 2 - 4 } & Absent & Present & Total \\
\hline Absent & 8 & 2 & 10 \\
Present & 4 & 4 & 8 \\
Total & 12 & 6 & 18
\end{tabular}

*Of the 12 patients without proliferative glomerulonephritis, six patients had normal histology, two had membranous glomerulopathy, three had non-specific abnormalities, and one had amyloidosis.

\section{Renal vasculitis}

Renal vasculitis was found in eight out of a total of 18 cases with systemic vasculitis at necropsy. Among these eight patients the glomeruli showed severe proliferative glomerulonephritis in four (see 'Glomerular lesions', below) and non-specific changes in one; the large renal vessels were normal in the other 10 . Two of these 10 patients, however, had severe proliferative glomerulonephritis, two had membranous glomerulopathy, two had nonspecific glomerular abnormalities, and one had amyloidosis. The results are summarised in Table 3.

\section{Glomerular lesions}

Glomerular lesions were found in 57 patients $(43 \%)$, with specific changes in 26 of them. Proliferative glomerulonephritis occurred in 11 patients: six had accompanying systemic vasculitis (see above). In these six, inflammation was severe, with extracapillary glomerulonephritis in four and diffuse

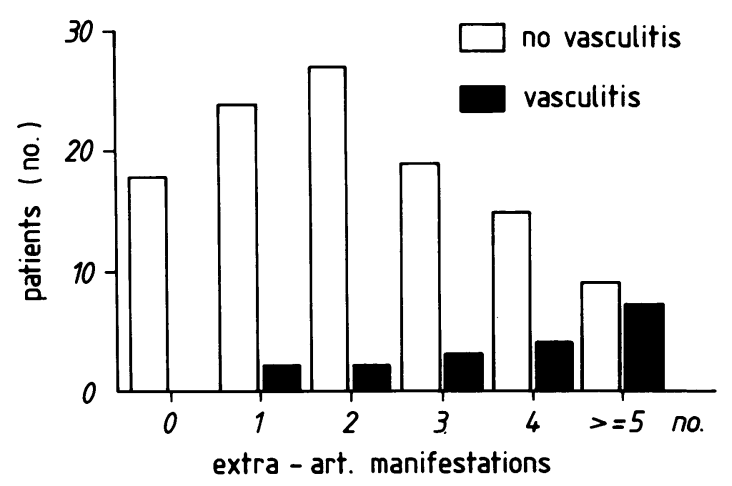

Fig. 1 Number of extra-articular manifestations (other than vasculitis) recorded before death in 132 patients with rheumatoid arthritis, grouped according to the presence or absence of vasculitis at necropsy. proliferative glomerulonephritis in two. In the five patients with proliferative glomerulonephritis but without systemic vasculitis, only one had diffuse $\Rightarrow$ glomerulonephritis, and four showed local focal glomerulonephritis.

Membranous glomerulopathy was observed in nine patients; in two, systemic vasculitis was found $\frac{\text { की }}{\frac{\rho}{\alpha}}$ at necropsy as well, but without evidence of renal $\stackrel{\mathbb{Q}}{\varrho}$ involvement. Focal glomerulosclerosis was noted in five patients (with concomitant amyloidosis in one), $\overrightarrow{0}$ and membranoproliferative glomerulonephritis in: one.

\section{Lesions of tubuli and interstitium}

Specific lesions of tubuli and interstitium were scarce; there were three patients with tubulointer-क stitial nephritis, and two with acute tubular necrosis.

\section{Benign nephrosclerosis}

Benign nephrosclerosis with characteristic changes윽 in glomeruli and larger renal vessels was found in $\vec{c}$ 119 patients $(90 \%)$; non-specific tubulointerstitial ${ }_{\mathbb{\Phi}}$ changes were found in 54 patients $(41 \%)$.

CLINICOPATHOLOGICAL CORRELATIONS Patients with amyloidosis had RA of longer duration $(\mathrm{p}<0.01)$ than patients without this finding. Mo over, proteinuria and uraemia were found moke frequently in the presence of amyloidosis; bo occurred in 11 of 14 patients (for both, $p<0.0017$.

The presence of systemic vasculitis (with or without renal involvement) at necropsy waso associated with the occurrence of other extra- $\frac{\circ}{\Phi}$ articular manifestations before death $(p<0 \cdot 01)$, as shown in Fig. 1. In patients with vasculitis, renaloㅡㅡ function loss occurred only where renal vessels were also affected, and uraemia only when proliferative glomerulonephritis was present (Fig. 2).

Only two of the five patients with proliferative glomerulonephritis but without systemic vasculitis:had renal function loss, and one other, the patient 3 . with diffuse glomerulonephritis, had uraemia. Fouri of the nine patients with membranous glomerulo-3 pathy had loss of renal function; in two of theseo four, renal function improved after the withdrawal of gold or D-penicillamine therapy. One patiento developed uraemia.

The three cases of interstitial nephritis wereo linked to clinical signs of pyelonephritis; one of these patients had recurrent ascending urinaryN్ infections and died of uraemia, and two patients had hydronephrosis with calculi; one of these two had renal function loss. The two cases of acute tubulare necrosis were associated with terminal catastrophice events (in once case gastrointestinal haemorrhage and in the other perforation peritonitis); both dief 5 before uraemia could develop. 


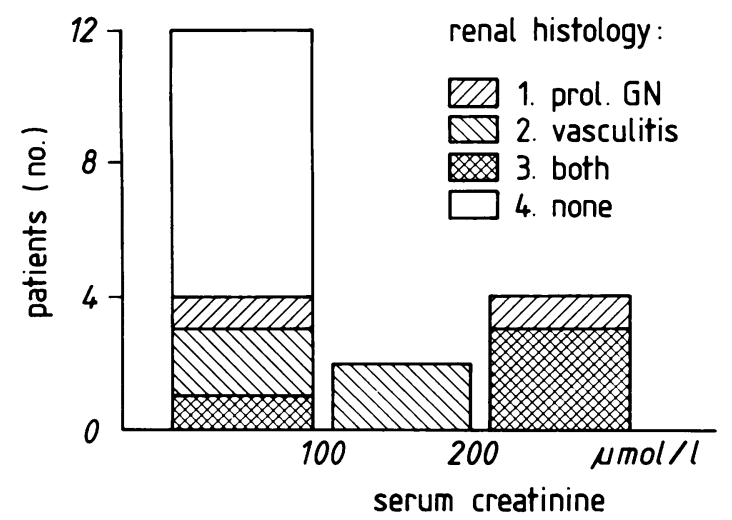

Fig. 2 Renal function at death $v$ renal histology in 18 patients with rheumatoid arthritis and systemic vasculitis at necropsy. (Prol. GN=proliferative glomerulonephritis).

Severity of benign nephrosclerosis was positively related to age $(p=0.05)$, duration of RA $(p<0.01)$, and occurrence of hypertension, arteriosclerosis, and/or diabetes (each, $\mathrm{p}<0.05)$, but not to renal function. Age and duration of RA were not interrelated $(p>0 \cdot 70)$. The presence of non-specific changes in tubuli and interstitium was positively related not only to the presence of hypertension $(\mathrm{p}<0.01)$, but also to the creatinine level during the

Table 4 Selected microscopic findings in the kidney, according to renal function at death in 132 necropsied patients with rheumatoid arthritis*

\begin{tabular}{lcccc}
\hline & \multicolumn{3}{l}{ Serum creatinine $(\mu m o l / l)$} \\
\cline { 2 - 5 } & Normal & 100 & 200 & Total \\
\hline Specific changes & & & & \\
in glomeruli & 4 & 2 & 5 & 11 \\
PGN & 7 & 1 & 1 & 9 \\
MGP & 0 & 1 & 0 & 1 \\
MPGN & 3 & 1 & 1 & 5 \\
FGS & & & & \\
& & & & \\
Non-specific changes in & & 5 & 12 & 39 \\
glomeruli and vessels & 22 & 15 & 12 & 67 \\
$\quad$ Absent/mild & 40 & 12 & 7 & 26 \\
$\quad$ Moderate & 7 & & & \\
Severe & & & & \\
Non-specific changes & & 4 & 11 & 19 \\
in tubulointerstitium & 62 & 26 & 15 & 103 \\
$\quad$ Absent/mild & 4 & 4 & 5 \\
$\quad \begin{array}{l}\text { Moderate } \\
\text { Severe }\end{array}$ & 1 & 0 & \\
\hline
\end{tabular}

${ }^{*}$ In some patients, specific and non-specific changes coincided. $\mathrm{PGN}=$ proliferative glomerulonephritis, $\mathrm{MGP}=$ membranous glomerulopathy; $\mathrm{MPGN}=$ membranoproliferative glomerulonephritis; FGS $=$ focal glomerulosclerosis. last year $(p<0 \cdot 01)$ and to the last recorded creatinine level $(p<0 \cdot 001)$.

The relations found between microscopic renal findings and renal function at death are summarised in Table 4.

No statistically significant relations, apart from those just mentioned, were found between a renal lesion and a given treatment modality or any other clinical parameter. It must be kept in mind, however, that all patients with systemic vasculitis and all those with membranous glomerulopathy had been treated with gold or D-penicillamine, or both.

\section{Discussion}

The results of this study show a high incidence of heterogeneous renal disease leading to uraemia in a group of necropsied patients with RA. Correlations with clinical data suggested that both RA and concomitant disease play a part in the aetiology of these lesions.

The prevalence of well known renal complications of RA, notably amyloidosis and membranous glomerulopathy, was similar to that reported by other authors. ${ }^{-+}$The relation between the presence of amyloidosis and the duration of RA, uraemia, and proteinuria was confirmed. Membranous glomerulopathy was found only in patients previously treated with gold or D-penicillamine, or both, but this relation was not statistically significant, probably owing to the large proportion of patients given such treatment. It must be remembered that some cases of membranous glomerulopathy will probably have been overlooked (in this and other necropsy studies) because immunofluorescence and electron microscopy techniques were not applied.

The proportion of our patients with evidence of systemic vasculitis at necropsy was high compared with the $2-5 \%$ prevalence in other reports. ${ }^{28}{ }^{29}$ The diagnosis vasculitis was often made only at necropsy and usually in patients with severe extra-articular disease of some duration. The evidence of renal damage found in half of the patients with systemic vasculitis is in line with reports of other series, in which renal abnormalities occurred in $20-40 \%$ of the patients with RA vasculitis, ${ }^{67}{ }^{30}$ and contradicts the assumption that renal involvement is rare in patients with this complication. ${ }^{28}$ In our patients the damage was frequently extensive and accompanied by severe exudative and proliferative glomerulonephritis leading to uraemia. In view of these findings systemic vasculitis and proliferative glomerulonephritis should be considered when renal abnormalities develop in a patient with many extraarticular manifestations of RA because early 
diagnosis of this form of glomerulonephritis followed by prompt immunosuppressive and cytostatic therapy improves the prognosis. ${ }^{31}$

The high incidence of benign nephrosclerosis in the present study can probably be ascribed mainly to concomitant disease. In a necropsy series of 150 'unselected' patients studied to assess the relation between hypertension and renal abnormalities, almost all of the patients had nephrosclerotic changes; the severity was strongly correlated with the presence of hypertension. ${ }^{32}$ In our patients the severity of the lesions was related to the presence of hypertension, diabetes mellitus, and arteriosclerosis. The finding of a clear relation between the severity of nephrosclerosis and the duration of RA remains unexplained, however, and suggests that RA or therapy is an additional factor in the aetiology of nephrosclerosis. We found no other non-specific renal abnormality, for example mesangial hypercellularity, that has been ascribed to RA. Subtle changes could easily have been 'hidden', however, among the nephrosclerotic lesions.

Our series is remarkable for the number of glomerular lesions not usually associated with RA, i.e., proliferative glomerulonephritis, focal glomerulosclerosis, and membranoproliferative glomerulonephritis. Proliferative glomerulonephritis has been reported incidentally in patients with $\mathrm{RA}^{14202133} 34$ and has been attributed to vasculitis and gold therapy. Six of our patients had glomerulonephritis associated with systemic vasculitis, but five had glomerulonephritis without evidence of vasculitis. Two of these five had not been treated with gold or D-penicillamine. To our knowledge membranoproliferative glomerulonephritis has not been reported before in patients with RA. In the necropsy study of 'normal' patients by Tracy and Toca $^{32}$ glomerulonephritis and focal sclerosis were found in less than $1 \%$. Thus the high incidence of glomerular lesions in our patients in comparison with these 'controls' suggests a previously unrecognised association of certain renal lesions with RA.

In summary, our study confirms the view that the kidney is frequently damaged in the course of a lifetime with RA. The scope of renal disease in RA encompasses well known entities such as amyloidosis, vasculitis, and membranous glomerulopathy, but may be wider and include other forms of glomerulopathy and benign nephrosclerosis. The associations between renal abnormalities and clinical data suggest that RA contributes to the renal damage caused by concomitant disease.

The authors would like to thank $G$ Brutel de la Riviere and $J$ Hermans for their contributions to this study and Ms A A J P Postma for secretarial assistance.
References

1 Glenner G G. Amyloid deposits and amyloidosis. $N$ Engl J Me 1980; 302: 1283-92, 1333-43.

2 Hall C L. Gold and D-penicillamine induced renal disease. It Bacon P A, Hadler N M, eds. The kidney and rheumatic disease London: Butterworth, 1982: 246-66.

3 Mutru O, Koota K. Isomäki H. Cause of death in autopsicd R patients. Scand J Rheumatol 1976; 5: 239-4().

4 Laakso M. Mutru O. Isomäki H, Koota K. Mortality from amyloidosis and renal diseases in patients with rheumatois arthritis. Ann Rheum Dis 1986; 45: 663-7.

5 Cove-Smith R. Analgesic nephropathy: clinical features and association with rheumatoid arthritis. In: Bacon P A. Hadler $\mathrm{M}$, eds. The kidney and rheumatic disease. London: Buttero worth, 1982: 228-45.

6 Schmid F R, Cooper N S, Ziff M. McEwan C. Arteritis in rheumatoid arthritis. Am J Med 1961; 30: 56-83.

7 Scott D G I, Bacon P A. Tribe C R. Systemic rheumatoi $\vec{P}$ vasculitis: a clinical and laboratory study of 50 cases. Medicine (Baltimore) 1981; 60: 288-97.

8 Bacon P A. Tribe C R. Mackenzie J C. Verrier Jones Jọ Cumming R H. Amer B. Penicillamine nephropathy in rheu을 matoid arthritis. $Q J$ Med 1976; 180: 661-84.

9 Baggenstoss A H. Rosenberg E F. Visceral lesions associate $\overrightarrow{0}$ with chronic infectious (rhcumatoid) arthritis. Archives od Pathology 1943; 35: 503-16.

10 Fingerman D L. Andrus F C. Visceral lesions associated wit $\frac{\mathbb{D}}{\square}$ rheumatoid arthritis. Ann Rheum Dis 1943: 3: 168-81.

11 Hordon L D. Sellars L. Morley A R. Wilkinson R, Thompsor巴 M. Griffiths I D. Haematuria in rheumatoid arthritis: an $^{2}$ association with mesangial glomerulonephritis. Ann Rheum $\Phi_{0} ;$ 1984; 43: 44()-3.

12 Lawson A A H, MacLean N. Renal disease and drug therap. rheumatoid arthritis. Ann Rheum Dis 1966; 25: 441-9.

13 Pasternack A, Wegelius D. Makisara P. Renal biopsy in rhcumatoid arthritis. Acta Med Scand 1967; 182: 591-5.

14 Ramirez G, Lambert R. Bloomer H A. Renal pathology ing patients with rheumatoid arthritis. Nephron 1981; 29: 124-6음

15 Salomon M I, Gallo G. Poon T P, Goldblat M V. Tchertkoff V尺 The kidney in rheumatoid arthritis. Nephron 1974; 12: 297-310 $\Rightarrow$ 16 Sellars L, Siamopoulos K. Wilkinson R. Leohapand T. Morles A R. Renal biopsy appearances in rheumatoid disease. Clin Nephrol 1983: 20: 114-20.

17 Brun C, Olsen T S, Raaschou F. Soerenson A W S. Renap biospy in rheumatoid arthritis. Nephron 1965; 2: 65-81.

18 Delaire M, Kahn M F. Morel-Maroger L. Méry J P, de Seze S Le rein dans le polyarthrite rhumatoide de l'adulte: étudé anatomique. Rev Rhum Mal Osteoartic 1974; 41: 105-11.

19 Mahallawy M N El. Sabour M S. Renal lesions in rheumatoid discase. Lancet 1959; ii: 852-3.

20 Davis J A, Cohen R A, Weisbart R, Paulus H E. Glomerulone phritis in rheumatoid arthritis. Arthritis Rheum 1979; 22윽

21 Friedman R F, Gallo G R, Buxbaum J N. Renal disease in rheumatoid arthritis. Arthritis Rheum 1980; 23: 781-3.

22 Samuels B, Lee J C, Engleman E P, Hopper J Jr. Membranous nephropathy in patients with rheumatoid arthritis: relationshipos to gold therapy. Medicine (Baltimore) 1977; 57: 319-27.

23 Pollak V E, Pirani C L, Steck I E, Kark R M. The kidney in rheumatoid arthritis: studies by renal biopsy. Arthritis Rheurf 1962; 5: $1-8$.

24 Allander E. Buch H, Lougren O, Wehle B. Renal function it rheumatoid arthritis. Acta Rheumatol Scand 1963; 8: 116-210

25 Burry H C. Reduced glomerular function in rhcumatoiō arthritis. Ann Rheum Dis 1972; 31: 65-8.

26 Camp A V, Mowat A G, Fletcher W J, Dunnill M S, McIver A G. A study of renal disease in rheumatoid arthritis. Ann Rheum Dis 1973; 32: 278-9. 
27 Churg J, Sobin L H. eds. Renal disease: classification and atlas of glomerular diseases. Tokyo: Igaku-Shoin Ltd, 1982.

28 Wilkinson D S. Rheumatoid vasculitis. In: Wolff K. Winkelmann R K. eds. Vasculitis. London: Lloyd-Luke Ltd, 1980: 188-202.

29 Meriwether J H Jr, Weinberger H J, Gleason I O. The renal lesion in rheumatoid disease [Abstract]. Arthritis Rheum 1967; 10: 298.

30 Kemper J W, Baggenstoss A H. Slocumb C H. The relationship of therapy with cortisone to the incidence of vascular lesions in rheumatoid arthritis. Ann Intern Med 1957; 46: 831-51.
31 Glassock R J. A clinical and immunopathologic dissection of rapidly progressive glomerulonephritis. Nephron 1978; 22: 253-64.

32 Tracy R E. Toca V T. Nephrosclerosis and blood pressure (parts I and II). Lab Invest 1974: 30: 20-34.

33 Breedveld F C, Valentijn R M. Westedt M L. Weening J J. Rapidly progressive glomerulonephritis with glomerular crescent formation in rheumatoid arthritis. Clin Rheumatol 1985: 4: 353-9.

34 Skrifvars B V, Tornroth T S, Tallquist G N. Gold induced immune complex nephritis in sero-negative rheumatoid arthritis. Ann Rheum Dis 1977; 36: 549-56. 